\title{
How much do patients on lithium know about their medication?
}

\author{
Norma Delany, Senior Registrar, Department of Psychiatry, Royal South Hants \\ Hospital, Graham Road, Southampton SO9 4PE (formerly Research Registrar, \\ University Department of Psychiatry, Royal South Hants Hospital)
}

Lithium is a drug used extensively in psychiatry for the treatment of depression, acute mania, and the prophylaxis of recurrent affective disorders. Lithium also has a place in the management of schizoaffective disorders and has been suggested for aggression and alcoholism. The side-effects, dangers of toxicity, and need for monitoring are well documented and should be considered before starting anyone on lithium. Likewise patients should be adequately informed of the benefits and hazards of treatment.

The aim of this study was to investigate how much patients on lithium know about various aspects of their medication. Understanding treatment is vital to encourage responsibility, to increase compliance, to minimise dangers and complications, and to enable patients to recognise side effects for what they are so that consent given to treatment is backed by adequate knowledge.

\section{The study}

The Psychiatry Out-patient Department at the Royal South Hants Hospital, Southampton, which serves a population of 290,000 , has approximately 250 patients on lithium recorded on a central register. All patients who saw the clinic sister to have their blood taken for a lithium level were requested to complete a two page self-administered questionnaire before they left the clinic. The patients were not given any assistance by the staff. Once 50 questionnaires were completed the study was ended. The questionnaire covered three areas:

(a) personal details: name, age, sex and occupation

(b) lithium treatment: dose, duration, believed diagnosis and whether they recall ever being given any written or verbal information on lithium

(c) knowledge of lithium: open questions were asked, "What do they test in your blood?" and "Do you know any effects of taking lithium for a long time?".

Thereafter a multiple choice format was used to assess what patients would do if they got "bad diarrhoea and vomiting" (dehydration), "forgot to take lithium tablets for two days", "a doctor suggested you should take water tablets (diuretics)", and "you got bad shaking of your hands, dizziness and vomiting" (suggesting toxicity). Patients were also asked to indicate from a list of symptoms which they would regard as side-effects which could be expected during treatment with lithium. Diagnosis and current dose of medication were compared with those obtained from medical notes.

As some measure of patients' knowledge, so that groups could be compared, each patient was given a score out of a total of 12 points for their factual knowledge of lithium.

\section{Findings}

Fifty people who were clinically stable completed the questionnaire. No-one refused to complete it. Thirteen $(26 \%)$ of those studied were 66 years or over. Twenty-eight $(56 \%)$ were not working outside the home, while $19(38 \%)$ had non-manual jobs and 3 $(6 \%)$ had manual work. The mean daily lithium dose of the sample was $687 \mathrm{mg}$ compared to $718 \mathrm{mg}$ of the whole lithium register (NS). Twenty-six (52\%) of the group studied were male compared with 210 (41\%) on the lithium register. The mean age of the sample was 53 years compared to 55 years of those on the register (NS). Six $(12 \%)$ patients had a casenote diagnosis of schizo-affective disorder, $11(22 \%)$ of bipolar affective disorder and the remainder, depression. Forty $(80 \%)$ patients correctly gave their case note diagnosis. One woman gave her disorder as purple sicosis (sic). Twelve (24\%) patients believed they were taking lithium because they were lithium deficient!

Fifteen (30\%) patients did not know why blood was taken. Of those who did know, $35(70 \%)$ knew a lithium level was measured, two (4\%) that kidney tests were done and three $(6 \%)$ that thyroid function was assessed. Most (94\%) were ignorant of possible long-term effects of treatment; others had misconceptions such as "sterility" and "memory damage". Two knew of possible thyroid changes and one of renal damage. 
In spite of the precautions patients need to take while on lithium, $16(32 \%)$ claimed never to have been given either verbal or written information. In addition, dangerous practices such as doubling the dose of lithium $(4 \%)$, taking diuretics $(24 \%)$, and ignoring situations leading to dehydration, such as sustained diarrhoea and vomiting $(44 \%)$, were common. Some patients had no understanding of lithium toxicity $(74 \%)$ or even what side-effects $(30 \%)$ to expect.

The score, an instrument used to assess global knowledge of lithium, had a mean value of 5.73 (out of a possible 12 points) with a standard deviation of 2.34. There was no significant difference in score when groups were divided according to sex, employment, diagnosis, duration of treatment and having received verbal or written information. However those over 65 years did have a significantly $(P<0.01)$ lower score $($ mean $=6.39, \mathrm{SE}=0.35)$ when compared with the younger group (mean $=3.85, \mathrm{SE}=0.54$ ). This is particularly worrying as the elderly are the group most likely to have physical illnesses, impaired renal function and problems with lithium.

\section{Comment}

Forgetting of information given to patients by doctors may in part account for the scant understanding of even those who had received either written or verbal information. Many patients take lithium for long periods so that the initial period of education and discussion of precautions and dangers of lithium may have been long forgotten. Ley (1982) reviewed 13 studies which assess patients' forgetting of material presented to them by doctors, and suggests the amount forgotten varies from $31-71 \%$. Forgetting was shown in some studies to be positively correlated with the amount of information given and age over 65 years. This may explain to some degree the particularly poor knowledge of the elderly, none of whom were obviously dementing.

Some clinicians may hesitate to discuss hazards or side-effects of drugs, fearing that this may reduce compliance. Indeed, compliance with long-term lithium medication leaves much room for improvement. Danion et al (1978) found that only $47 \%$ of 68 patients were thought to be complying perfectly. However Myers \& Calvert (1973) have shown that forewarning patients of the side-effects of two antidepressant drugs did not increase the incidence of reported side-effects or significantly influence the rate of discontinuation of medication. In further correspondence (Myers \& Calvert, 1978) patients given both written and verbal information discontinued their medication significantly less often than those given no information or only verbal information.

Ley et al (1976) have emphasised the importance of patients' understanding the instructions given to them. They found that compliance was improved by appropriate leaflets but only when they were comprehensible to the patients as measured by the Flesch Formula. In this study, although $50 \%$ of the sample group admitted to having been given written information this does not appear to have significantly improved their understanding of their treatment as measured by their score, when compared to those given no information or just verbal information. This may be related to the complexity of the information given. The leaflet used in the clinic is eight pages long and tends to have a discursive style rather than clear do's and don'ts.

In conclusion, it appears from this study that patients on lithium know dangerously little about their medication; this may be due to clinicians' reluctance to inform them, patients forgetting or the inadequacy of the written literature. Compliance and safety could well be improved by repeated, simple verbal and written information.

\section{Acknowledgements}

The author wishes to thank Professor Chris Thompson who supervised the project and Mrs E. King for statistical assistance.

\section{References}

Danion, J. M., Neunreuther, C., Krieger-Finance, F. et al (1978) Compliance with long-term treatment in major affective disorders. Pharmacopsychiatrica, 20, 230-231.

LeY, P., Jain, V. K. \& SilbeCK, C. E. (1976) A method for decreasing patients' medication errors. Psychological Medicine, 6, 599-601.

(1982) Satisfaction, compliance and communication. British Journal of Clinical Psychology, 21, 241-254.

MYERS, E. D. \& CALVERT, E. J. (1973) The effect of forewarning on the occurrence of side-effects and discontinuation of medication in patients on amitryptyline. British Journal of Psychiatry, 122, 461-464.

\& \& (1978) Knowledge of side-effects and perseverance with medication. British Journal of Psychiatry, 132, 526-527. 